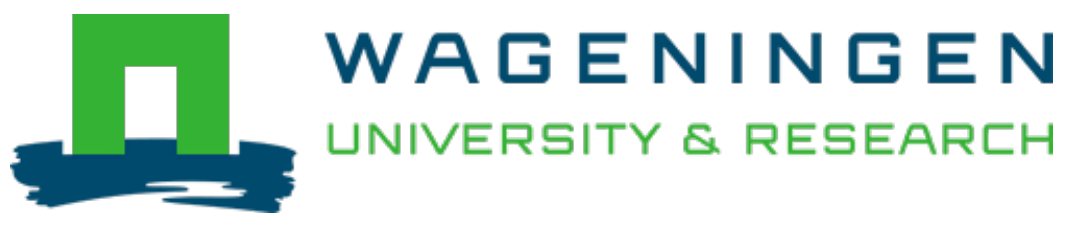

\title{
Reductive Glycine Pathway : A Versatile Route for One-Carbon Biotech
}

Trends in Biotechnology

Claassens, Nico J.

https://doi.org/10.1016/j.tibtech.2021.02.005

This article is made publicly available in the institutional repository of Wageningen University and Research, under the terms of article $25 \mathrm{fa}$ of the Dutch Copyright Act, also known as the Amendment Taverne. This has been done with explicit consent by the author.

Article $25 \mathrm{fa}$ states that the author of a short scientific work funded either wholly or partially by Dutch public funds is entitled to make that work publicly available for no consideration following a reasonable period of time after the work was first published, provided that clear reference is made to the source of the first publication of the work.

This publication is distributed under The Association of Universities in the Netherlands (VSNU) 'Article 25fa implementation' project. In this project research outputs of researchers employed by Dutch Universities that comply with the legal requirements of Article $25 \mathrm{fa}$ of the Dutch Copyright Act are distributed online and free of cost or other barriers in institutional repositories. Research outputs are distributed six months after their first online publication in the original published version and with proper attribution to the source of the original publication.

You are permitted to download and use the publication for personal purposes. All rights remain with the author(s) and / or copyright owner(s) of this work. Any use of the publication or parts of it other than authorised under article $25 \mathrm{fa}$ of the Dutch Copyright act is prohibited. Wageningen University \& Research and the author(s) of this publication shall not be held responsible or liable for any damages resulting from your (re)use of this publication.

For questions regarding the public availability of this article please contact openscience.library@,wur.nl 
cultures. Novel strategies to control the ecosystem inside reactors and cope with outdoor conditions must be developed at the strain engineering stage, hand-in-hand with optimization of scaleup cultivation. To develop managed stable cultivation systems, synthetic consortia [13], or media/strain systems that exclude growth of other organisms should be systematically evaluated [14]. Robust thermophilic and halophilic strains such as Synechococcus PCC 7002 may be less prone to contamination and could be ideal hosts for future engineering efforts that are amenable to scale.

The holistic approach of PHOTOFUEL towards engineered fuel secretion from light and $\mathrm{CO}_{2}$ enabled a targeted and critical discourse to steer engineering efforts at all levels. Genetic engineers took feedback from industrial culture experts, who interacted with process design specialists to develop cultivation strategies for secreted hydrocarbons. Engine testing and technological challenges in product separation from aqueous cultures influenced genetic engineering strategies, and market and perception analysis impacted business case and scale planning. Although comprehensive studies are needed to identify optimal systems for cost-effective commercial biomanufacturing of fuels, PHOTOFUEL has set the foundation for their development and outlined some of the key issues to be addressed in the future.

\section{Acknowledgments}

The authors would like to thank Dr. Michael Bippes for scientific advice regarding engine tests. This work was supported by the EU Horizon 2020 research and innovation program Photofuel (grant No 640720).

${ }^{1}$ Bielefeld University, Faculty of Biology, Center for Biotechnology (CeBiTec), Universitätsstrasse 27, 33615 , Bielefeld, Germany

${ }^{2}$ University of Florence, Department of Agriculture, Food, Environment, and Forestry (DAGRI), Piazzale delle Cascine 18, 50144 Florence, Italy

${ }^{3}$ Volvo Group Trucks Technology, Sven Hultins gata 9A, 41288 Gothenburg, Sweden
${ }^{4}$ A4F, Algae For Future, Campus do Lumiar, Estrada do Paço do Lumiar Ed E R/C, 1649-038 Lisboa, Portugal 5 Imperial College London, Department of Chemical Engineering, South Kensington, SW7 2AZ London, UK ${ }^{6}$ SYNCOM RTD Consulting, Am Steinacker 16, 27777 Ganderkesee, Germany

${ }^{7}$ Neste Corporation, Keilaranta 21, 02150 Espoo, Finland ${ }^{8}$ Uppsala University, Microbial Chemistry, Department of Chemistry-Ångström, Box 523, SE-751 20 Uppsala, Sweden ${ }^{9}$ Karlsruhe Institute of Technology (KIT), Institute for Technology Assessment and Systems Analysis (ITAS), Karlstr.11, 76133 Karlsruhe, Germany

${ }^{10}$ Imperial College London, Department of Life Sciences, SW7 2AZ London, UK

\section{${ }^{*}$ Correspondence:}

olaf.kruse@uni-bielefeld.de (O. Kruse).

https://doi.org/10.1016/j.tibtech.2021.01.003

C 2021 Elsevier Ltd. All rights reserved.

\section{References}

1. Miao, R. et al. (2018) Enhancement of photosynthetic isobutanol production in engineered cells of Synechocystis PCC 6803. Biotechnol. Biofuels 11, 1-9

2. Miao, R. et al. (2018) Protein engineering of $a$ ketoisovalerate decarboxylase for improved isobutano production in Synechocystis PCC 6803. Metab. Eng. 47, $42-48$

3. Miao, R. et al. (2017) Isobutanol production in Synechocystis PCC 6803 using heterologous and endogenous alcohol dehydrogenases. Metab. Eng. Commun. 5 , 45-53

4. Liu, X. et al. (2019) Modular engineering for efficient photosynthetic biosynthesis of 1-butanol from CO2 in cyanobacteria. Energy Environ. Sci. 12, 2765-2777

5. Yunus, I.S. and Jones, P.R. (2018) Photosynthesisdependent biosynthesis of medium chain-length fatty acids and alcohols. Metab. Eng. 49, 59-68

6. Sattayawat, P. et al. (2020) Bioderivatization as a concept for renewable production of chemicals that are toxic or poorly soluble in the liquid phase. Proc. Natl. Acad. Sci. U. S. A. 117, 1404-1413

7. Yunus, I.S. et al. (2020) Methanol-free biosynthesis of fatty acid methyl ester (FAME) in Synechocystis sp. PCC 6803 Metab. Eng. 57, 217-227

8. Yunus, I. et al. (2018) Synthetic metabolic pathways for photobiological conversion of $\mathrm{CO}_{2}$ into hydrocarbon fuel. Metab. Eng. 49, 201-211

9. Wichmann, J. et al. (2018) Tailored carbon partitioning for phototrophic production of $(E)$-a-bisabolene from the green microalga Chlamydomonas reinhardtii. Metab. Eng. 45, 211-222

10. Miao, R. et al. (2020) Current processes and future challenges of photoautotrophic production of acetyl-CoAderived solar fuels and chemicals in cyanobacteria. Curr. Opin. Chem. Biol. 59, 69-76

11. Nilsson, A. et al. (2020) Environmental impacts and limitations of third-generation biobutanol: life cycle assessment of n-butanol produced by genetically engineered cyanobacteria. J. Ind. Ecol. 24, 205-216

12. Varela Villarreal, J. et al. (2020) Acceptability of genetically engineered algae biofuels in Europe: opinions of experts and stakeholders. Biotechnol. Biofuels 13, $1-21$

13. Kazamia, E. et al. (2012) Synthetic ecology - a way forward for sustainable algal biofuel production? J. Biotechnol. 162, 163-169

14. Loera-Quezada, M.M. et al. (2016) A novel genetic engineering platform for the effective management of biologica contaminants for the production of microalgae. Plant Biotechnol. J. 14, 2066-2076
Special Issue: Bioconversion of $\mathrm{C} 1$ Products and Feedstocks

Spotlight

\section{Reductive Glycine Pathway: A Versatile Route for One- Carbon Biotech}

Nico J. Claassens ${ }^{1, *, @}$

Hong et al. heterologously expressed the metabolic core of the reductive glycine pathway (rGlyP) as a sink for the anaerobic conversion of glycerol. This recent study concludes several reports in 2020 on the ATP-efficient, one-carbonassimilating rGlyP. Its engineering in diverse hosts could help the transformation toward renewable, one-carbon-based

bioproduction.

The reductive glycine pathway (rGlyP) is a synthetic pathway for formate assimilation [1]. This soluble one-carbon molecule has been suggested as a promising, sustainable substrate for future biotechnology [2]. In rGlyP, formate is first activated with the co-factor tetrahydrofolate into formylTHF, at the expense of one ATP (Figure 1A). Next, formyl-THF is reduced to methylene-THF, which is then condensed and reduced with $\mathrm{CO}_{2}, \mathrm{NH}_{3}$, and $\mathrm{NADH}$ into glycine. This formation of glycine is catalyzed by the glycine cleavage/synthase system, a reversible four-component enzyme, which can catalyze the synthesis of glycine under elevated $\mathrm{CO}_{2}$ concentrations. The overall conversion of formate and $\mathrm{CO}_{2}$ into glycine can be defined as the core module of rGlyP. Glycine can be further assimilated into biomass and product precursors via several routes, such as by the 
(A)
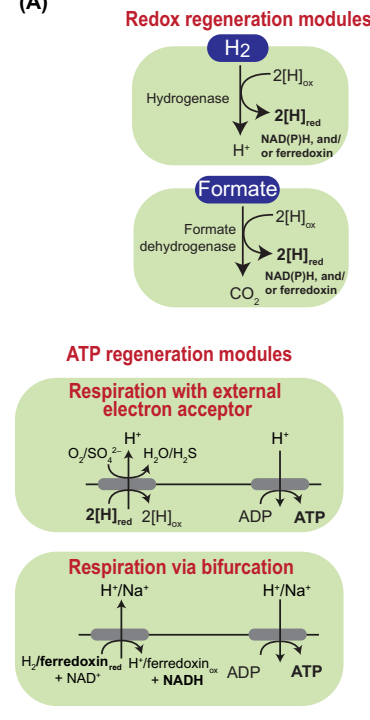

(B)

Reductive glycine pathway as sink for anaerobic conversion

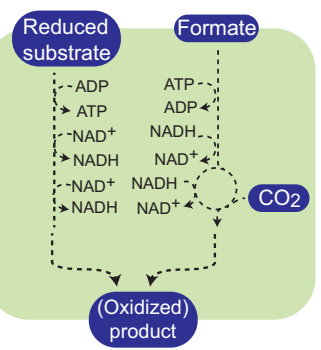

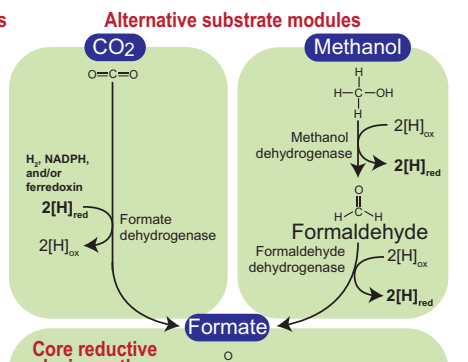

Alternative substrate modules
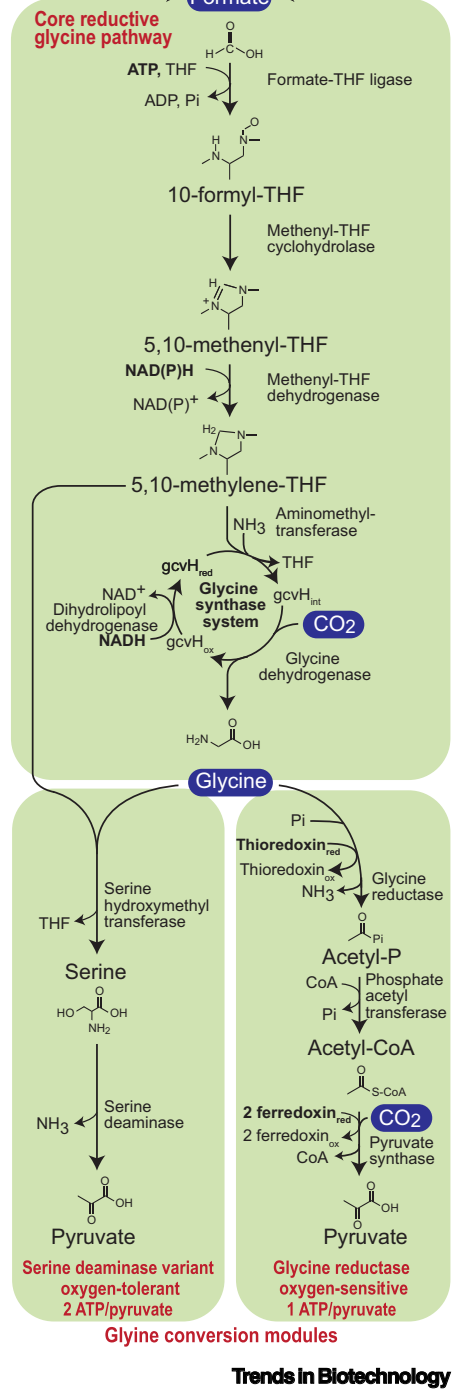

Figure 1. Metabolic Scheme of Reductive Glycine Pathway and Related Modules. (A) The core metabolic module of the reductive glycine pathway (formate to glycine) and additional metabolic modules for energy supply, the conversion of alternative substrates to formate, and the conversion of glycine to the central metabolic intermediate pyruvate. Note that more modules for glycine conversion to pyruvate can be envisioned with varying ATP costs, such as a 'wasteful' variant observed in Desulfovibrio desulfuricans for the fixation of $\mathrm{CO}_{2}$ via the pathway [7]. In this case, acetyl-phosphate formed by glycine reductase is first converted to acetate by a kinase and, subsequently, to acetyl-CoA by acetyl-CoA synthetase, costing overall 2 ATP/pyruvate. (B) Simplified metabolic scheme depicting the reductive glycine pathway as a sink for electrons during the anaerobic oxidation of a reduced substrate, such as the fermentation of glycerol, as proposed by Hong et al. [10]. for formate assimilation, including full pathway implementation in the bioplasticproducer Cupriavidus necator [5], and demonstration of the core module of rGlyP from formate to glycine in Saccharomyces cerevisiae [6].

Another recent study showed that a variant of rGlyP can operate as a natural $\mathrm{CO}_{2}$ fixation route in Desulfovibrio desulfuricans G11, which grows autotrophically on hydrogen in anaerobic conditions (via glycine reductase) [7]. Here, $\mathrm{CO}_{2}$ is first reduced to formate via the reversible formate dehydrogenase. This study confirmed the rGlyP as the seventh $\mathrm{CO}_{2}$ fixation pathway known in nature, after a previous study suggested this based on metagenomics [8]. Among the known $\mathrm{CO}_{2}$ fixation routes, rGlyP is also one of the most 
ATP-efficient pathways, only rivalled again by the Wood-Ljungdahl pathway and the reductive tricarboxylic acid cycle, which also both require $\sim 1-2$ ATP/pyruvate.

The low ATP costs of the rGlyP also make it a potentially promising production route for anaerobic industrial conditions, in which ATP is more limited. However, in anaerobic conditions, ATP is still required to drive rGlyP. In anaerobic $\mathrm{CO}_{2}$ fixation via rGlyP by $D$. desulfuricans, ATP is regenerated via respiration with sulfate as an electron acceptor. However, for most anaerobic biotech processes, the use of limitedly available electron acceptors, such as sulfate or nitrate, is undesired. Hence, ATP-regeneration mechanisms other than anaerobic respiration are required. Anaerobic acetogens utilizing the Wood-Ljungdahl pathway operate energy-conserving mechanisms to produce ATP via the activity of bifurcating enzymes. Some bifurcating respiration enzymes can generate an ion gradient across the cell membrane to drive ATP synthase, using the energetic difference between higher and lower potential redox carriers (e.g., ferredoxin and NADH). The recent discovery of rGlyP operating in parallel to the Wood-Ljungdahl pathway in the acetogen Clostridium drakei, as well as the functional heterologous expression of rGlyP into the acetogen Eubacterium limosum, suggest that these bifurcation mechanisms can also support rGlyP [9]. Both the WoodLjungdahl pathway and rGlyP can allow for highly efficient anaerobic conversion of formate (or $\mathrm{H}_{2} / \mathrm{CO}_{2}$ ) into products such as acetate, ethanol, and butanol. However, many products cannot be made using these pathways under anaerobic conditions, because too little ATP is available for their biosynthesis.

A recent study by Hong and colleagues [10] proposes an alternative application for rGlyP in anaerobic conditions. They suggest rGlyP as a sink for electrons under anaerobic conditions when a highly reduced substrate, such as glycerol, is used (Figure 1B). In fact, during the 1980s, the role of rGlyP as an electron sink was described in anaerobic degraders of highly reduced purines [11]. A potential biotechnological application could be the co-fermentation of glycerol with formate in a bacterium harboring rGlyP to enable efficient conversion into products that are more oxidized than glycerol. On the road to establish the rGlyP in an anaerobic, glycerol-fermenting bacterium, Hong and colleagues equipped Clostridium pasteurianum with the missing glycine synthase system by expressing from a plasmid the four genes encoding the glycine synthase system from the purine-degrader Gottschalkia acidurici. Unfortunately, the heterologous expression of these genes caused a high burden, eliminating growth of this organism on glycerol and formate. Still, the authors observed increased uptake of formate, and some physiological impacts of formate addition in the presence of glucose, suggesting that the heterologous glycine synthase system is active. Thus, further studies are needed to optimize the expression of this enzyme complex and the rest of the pathway in C. pasteurianum. Another recent study in which the rGlyP was established by engineering and lab evolution in $C$. necator showed that proper balancing of the expression of the glycine synthase system is crucial to achieve sufficient rGlyP activity, while preventing a detrimental expression burden of this system [5]. I expect that further engineering of rGlyP, including improved understanding and optimization of the glycine synthase system kinetics [12], will enable future sustainable biotech applications using various one-carbon substrates in diverse hosts and conditions.

\section{Acknowledgments}

I am grateful to Arren Bar-Even (1980-2020) for inspiring and supporting me and many others to work on the reductive glycine pathway. I thank Ari Satanowski and Steffen Lindner for critical reading of this manuscript. I acknowledge support from a Veni Fellowship of the Dutch Science Organisation (NWO) (VI.Veni.192.156).

\section{Declaration of Interests} None declared by author.

1'Laboratory of Microbiology, Wageningen University, Stippeneng 4, 6708, WE, Wageningen, The Netherlands

${ }^{*}$ Correspondence:

nico.claassens@wur.nl (N.J. Claassens).

@Twitter: @NicoC_MicSynBio, @MIB_WUR (N.J. Claassens). https://doi.org/10.1016/j.tibtech.2021.02.005

๑) 2021 Elsevier Ltd. All rights reserved.

References

1. Bar-Even, A. et al. (2013) Design and analysis of metabolic pathways supporting formatotrophic growth for electricitydependent cultivation of microbes. Biochim. Biophys. Acta Bioenerg. 1827, 1039-1047

2. Yishai, O. et al. (2016) The formate bio-economy. Curr. Opin. Chem. Biol. 35, 1-9

3. Cotton, C.A.R. et al. (2020) Renewable methanol and formate as microbial feedstocks. Curr. Opin. Biotechnol. 62, $168-180$

4. Kim, S. et al. (2020) Growth of E. coli on formate and methanol via the reductive glycine pathway. Nat. Chem. Biol. 16, 538-545

5. Claassens, N.J. et al. (2020) Replacing the Calvin cycle with the reductive glycine pathway in Cupriavidus necator. Metab. Eng. 62, 30-41

6. Gonzalez, J. et al. (2019) Core catalysis of the reductive glycine pathway demonstrated in yeast. ACS Synth. Biol. 8, 911-917

7. Sánchez-Andrea, I. et al. (2020) The reductive glycine pathway allows autotrophic growth of Desulfovibrio desulfuricans. Nat. Commun. 11, 1-12

8. Figueroa, I.A. et al. (2017) Metagenomics-guided analysis of microbial chemolithoautotrophic phosphite oxidation yields evidence of a seventh natural $\mathrm{CO} 2$ fixation pathway. Proc. Natl. Acad. Sci. U. S. A. 115, E92-E101

9. Song, Y. et al. (2020) Functional cooperation of the glycine synthase reductase and Wood-Ljungdahl pathways for autotrophic growth of Clostridium drakei. Proc. Natl. Acad. Sci. U. S. A. 117, 7516-7523

10. Hong, Y. et al. (2020) Introduction of glycine synthase enables uptake of exogenous formate and strongly impacts the metabolism in Clostridium pasteurianum. Biotechnol. Bioeng. Published online December 17, 2020. https://doi.org/10.1002/bit.27658

11. Durre, P. and Andreesen, J.R. (1982) Pathway of carbon dioxide reduction to acetate without a net energy requirement in Clostridium purinolyticum. FEMS Microbiol. Lett. $15,51-56$

12. Zhang, H. et al. (2020) Structure-based dynamic analysis of the glycine cleavage system suggests key residues for control of a key reaction step. Commun. Biol. 3, 1-12 